García Hernández, J.L., Cortés Pascual, A. (2020). ¿Qué propone la orientación profesional ante las políticas de empleo de la España postcrisis? Un análisis cualitativo. Revista de Investigación Educativa, 38(1), 89-108. DOI: http://dx.doi.org/10.6018/rie.324771

\title{
¿Qué propone la orientación profesional ante las políticas de empleo de la España postcrisis? Un análisis cualitativo
}

\section{What proposes vocational guidance to employment policies of Spain postcrisis? A qualitative analysis.}

\author{
José Luis García Hernández* y Alejandra Cortés Pascual** \\ *Departamento Educación Gobierno de Navarra. (España) \\ **Departamento Ciencias de la Educación. Universidad de Zaragoza. (España)
}

\begin{abstract}
Resumen
La orientación es el primer eje de las políticas activas de empleo de la Estrategia Española de Activación para el Empleo. Esta disciplina presenta un claro posicionamiento teórico-formal justificado por el influjo de las directrices y modelos europeos, y por toda una red de normas educativas, formativas y sociolaborales. Así mismo, la coyuntura actual sitúa a la orientación ante el reto de aportar soluciones y mejorar la dinamización de las políticas de empleo. Estos retos y realidades en el marco sociolaboral se complejizan aún más ante la observación del terreno de actuación práctica real, condicionada por los efectos de la última crisis económica, que refleja un escenario de políticas, sistemas, estructuras y servicios de orientación con evidentes áreas de mejora. A partir de un diseño de Teoría Fundamentada, alineado con el paradigma cualitativo/interpretativo y los fundamentos del interaccionismo simbólico, se analizó a través de entrevistas semiestructuradas toda una diversidad de voces autorizadas, tanto a nivel nacional como internacional, emergiendo un abanico de temas que dibujan el terreno actual de la orientación en las próximas décadas. Los resultados describen las debilidades, amenazas, fortalezas y oportunidades de nuestro sistema, especialmente en la compleja última década. Se concluye con la confianza en un futuro mejor, que pasa obligatoriamente por afrontar los retos y debilidades que emergen de esta investigación, así como el reforzamiento de las dinámicas más positivas que se observan en el sistema de orientación.
\end{abstract}

Correspondencia: Alejandra Cortés Pascual. alcortes@unizar.es. Departamento Ciencias Educación. UNIZAR. C/ Pedro Cerbuna, no 12. 50009 Zaragoza. 
Palabras claves: Orientación profesional; investigación cualitativa; política del empleo; flexibilidad; crisis económica; España.

\begin{abstract}
Orientation is the first axis of active employment policies, structured in the Spanish Activation Strategy for Employment 2014-2016. This scientific discipline presents a clear theoretical and formal position, justified by the influence of European guidelines and models, and by a myriad of educational, training and socio-labor standards. Likewise, the present and future situation places professional orientation in the face of the challenge of providing solutions and improving the dynamisation of employment policies. These remains and realities that show the orientation in the socio-labor framework, are further complicated by the observation of the field of practical and real action, a territory conditioned by the effects of the last economic crisis, which draw a scenario of policies, systems, structures and services of professional orientation with evident areas of improvement. Based on a design of Grounded Theory, aligned with the qualitative / interpretative paradigm and the foundations of symbolic interactionism, a semistructured interviews were analyzed through a variety of authorized voices, both nationally and internationally, emerging a range of themes that draw the current ground of orientation in the coming decades. The results describe the weaknesses, threats, strengths and opportunities of our system, especially in the complex last decade. It concludes with confidence in a better future, which necessarily involves facing the challenges and weaknesses that emerge from this research, as well as reinforcing the most positive dynamics observed in the guidance system.

Keywords: Career guidance; qualitative research; employment policy; flexibility; economic recession; Spain.
\end{abstract}

\title{
Introducción
}

La orientación está alcanzando un reconocimiento importante en las leyes laborales. Su posicionamiento oficial como primer eje de políticas activas de empleo es evidente, tal y como se muestra en el Real Decreto 1032/2017, de 15 de diciembre, por el que se aprueba la EEAE 2017-2020. Pero su desarrollo real práctico, junto a las dudas sobre las pruebas científicas que demuestren su impacto, contrasta con nuestro panorama de relaciones laborales, caracterizado por una significativa problemática de acceso y mantenimiento en el empleo, junto a una cuestionable calidad del mismo para amplias capas de nuestra sociedad (Torres, 2015).

Varios aspectos han favorecido el aumento del peso político de la orientación, situándola como una disciplina a desarrollar por los responsables políticos (Organización para la Cooperación y el Desarrollo Económicos, 2004). Primeramente, la centralidad del desarrollo de la empleabilidad, planteada por la Unión Europea no como creación de empleo directo, sino como la mejora de las condiciones de acceso y promoción en el empleo (García-Hernández \& Cortes, 2019). Este concepto sitúa a la orientación como su elemento facilitador junto a otras políticas activas como la formación o la intermediación laboral. Empleabilidad que subraya la importancia de la responsabilidad individual como forma de adaptación a las cambiantes exigencias del mercado laboral. 
En segundo lugar, el paradigma de flexiseguridad, que sitúa a la orientación en esa pluralidad de dispositivos que tejen una teórica red de nueva seguridad ante las dinámicas de los mercados de trabajo transicionales (Wilthagen \& Tros, 2004), poniendo el acento no tanto en la seguridad tradicional del periodo fordista, sino más bien la seguridad en el empleo en general (Fernández \& Serrano, 2014). En tercer lugar, la orientación a lo largo de la vida, derivada del aprendizaje a lo largo de la vida, clave en la sociedad del conocimiento, que considera especialmente valiosa la mejora competencial como forma de gestionar mejor la carrera (Sánchez García, 2017).

De esta forma, la orientación trata de conectar los mundos de la educación formal, el empleo y la formación para el empleo, planteando una mejor gestión de carreras y competencias (Echeverría, 2016; Sobrado \& Cortés, 2009). Pero estas funciones de la orientación se vienen desarrollando en un contexto que plantea unos estados de cuestionamiento e inestabilidad constante de los desarrollos de carrera (Corominas, 2006). Todo ese andamiaje teórico-normativo contrasta claramente con los efectos de la última crisis económica, que parece haber superado claramente las posibilidades de prevención y respuesta de nuestro sistema de orientación.

Comparando el momento pre-crisis con la actualidad, se muestra según los datos de la Encuesta de Población Activa (IV trimestre), una bajada de la tasa de actividad (2007-2018) de 0.86 puntos, con grandes cambios por franjas de edad (subiendo en veteranos, y disminuyendo en jóvenes). Una población ocupada que disminuyó durante 6 años (2008-2014) destruyéndose 3.8 millones de empleos, generándose posteriormente 2 millones (2014-2018; con fuertes transformaciones internas (menos jornadas completas, y más jornadas parciales). Una tasa de desempleo que pasó del 7.9\% al 26.9\% (20072013), bajando al 14.5\% al final de 2018. Dibujando distintos escenarios en materia de desempleo y temporalidad dentro del mismo país.

Cabe indicar que nuestro sistema de orientación se enmarca en unas políticas de empleo que son las que mayor porcentaje de PIB protagonizan de la UE, con un 3.3\%. Este buen posicionamiento cambia al medir la relación gasto en políticas de empleo/ punto tasa de desempleo, ya que España con un $0.12 \%$ presenta un gasto muy inferior a la media europea. Dentro de las políticas de empleo, las políticas activas suponen un $0.41 \%$ del PIB español, inferior a la media europea, y muy alejado de países como Dinamarca con un $1.42 \%$. Especialmente llamativo es el peso de las políticas de orientación en las políticas activas, que protagonizan un 15\% en España, en comparación con el 30\% de media de la UE. Con la singularidad de que España es quien más peso dedica al fomento del emprendimiento en Europa, un 20\% de sus políticas activas, en comparación con la media del 5\% (Consejo Económico y Social, 2016). Así mismo, diversos estudios sobre los sistemas de orientación autonómicos vienen señalando su débil articulación (García-Hernández \& Sánchez-Santamaría, 2013).

Para tratar de dar respuesta a este planteamiento del problema de investigación, el enfoque metodológico desarrollado ha sido netamente cualitativo, escogiendo el diseño de Teoría Fundamentada (Grounded Theory) como más adecuado por sus planteamientos alineados con el paradigma cualitativo/interpretativo y los fundamentos del interaccionismo simbólico. Diseño que se dota de un procedimiento sistemático cualitativo para generar una teoría explicativa de un área específica (Glaser, 2014). 


\section{Método}

Se ha optado por una investigación cualitativa, con un enfoque dirigido a los puntos de vista subjetivos, a través de un diseño de teoría fundamentada. Con el método de recogida de datos de entrevista semiestructurada. La finalidad de esta investigación ha sido analizar el estado de opinión del ecosistema de personas expertas en orientación y el empleo.

\section{Objetivos}

La investigación se ha fijado como objetivo general el analizar el papel de la orientación profesional en las actuales políticas de empleo, y como objetivos específicos: 1) Entender las razones por las que la orientación profesional adquiere un papel estratégico en las actuales políticas de empleo; 2) Entender las limitaciones, contradicciones, ambivalencias de la orientación ante las dinámicas y retos del mercado laboral español; 3) Explorar el grado de integración que se experimenta en torno a la orientación profesional; 4) Analizar los discursos en torno al planteamiento y cumplimiento de los objetivos fijados para la orientación en materia de política de empleo (García-Hernández, 2019.

\section{Muestra}

La muestra final se compone de 52 personas expertas, (17\% internacionales, $45 \%$ nacionales, y 38\% autonómico). Una muestra inicialmente flexible en su composición numérica, pero diversa, para poder captar los discursos de expertos en diversos ámbitos: academia; agentes sociales; organismos internacionales; tercer sector; y Administración (Neuman, 2009).

Se contactó con las personas expertas a través de una carta; y tras la respuesta afirmativa se agendaba la forma de realización de la entrevista semi-estructurada (presencial o Skype), comunicando el interés en grabar el audio de esta para su posterior análisis, siempre garantizando el carácter anónimo de las opiniones (Alcadipani \& Hogdson, 2009). No se realizaron más de 52 entrevistas (Ver Tabla 1) al considerar el investigador saturado el discurso (Hernández, Fernández \& Baptista, 2010).

Tabla 1

Listado de personas entrevistadas.

\section{Organización. Cargo de la persona. Ubicación}

Organización Internacional del Trabajo OIT. Economist Research Department. Ginebra (Suiza).

Centro Europeo para el Desarrollo de la Formación Profesional CEDEFOP. Project manager on lifelong guidance. Tesalónica (Grecia).

CEDEFOP, y Fundación Tripartita para la Formación en el Empleo. Representante. Madrid. 


\section{Organización. Cargo de la persona. Ubicación}

European Lifelong Guidance Policy Network ELGPN representante español. Jefe de la Subdirección General de Orientación y Formación Profesional. Ministerio de Educación, Cultura y Deporte. Madrid.

Ministerio de Educación, Cultura y Deporte. Madrid. Ex-representante de la Dirección General de Formación Profesional

Universidad Complutense de Madrid. Profesora Departamento de Psicología Social. Ex- Investigadora en European Trade Union Institute - Confederación Europea de Sindicatos.

Investigador experto en formación profesional y políticas de empleo. Colaborador de: CEDEFOP, Fundación Europea para la Formación y gobiernos estatales y regionales. Barcelona.

Fondo Social Europeo. Representante Directorate-General for Employment, Social Affairs and Inclusion. Bruselas.

Comisión Europea. Directorate-General for Economic and Financial Affairs. Unit Economies of the Member States. Responsable Gestión seguimiento reformas y Semestre Europeo España. Bruselas.

Universidad Autónoma de Madrid. Profesor departamento de sociología.

Universidad Autónoma de Barcelona. Profesor Departamento sociología. Miembro Centre d'estudis sociològics sobre la vida quotidiana i el treball QUIT, e Instituto Estudios del Trabajo IET. Barcelona.

Universidad de Barcelona. Catedrático Emérito de Métodos de Investigación y Diagnóstico en Educación. Barcelona.

Universidad Nacional de Educación a Distancia Profesora Departamento Métodos de Investigación y Diagnóstico en Educación II. Miembro del Consejo Asesor Nacional Revista Española de Orientación y Psicopedagogía. Madrid.

Universidad de Zaragoza. Profesora Departamento Métodos de Investigación y Diagnóstico, en Educación Zaragoza.

Universidad de Salamanca. Profesor Departamento de Economía e Historia Económica.

Universidad de Oviedo. Profesora Departamento de Economía Aplicada. Miembro de la Asociación Española de Economía del Trabajo. Oviedo.

Asociación Navarra de Empresa Laborales. Técnico de emprendimiento. Pamplona.

Universidad Pública de Navarra. Profesora Departamento de Trabajo Social. Pamplona.

Universidad del País Vasco. Catedrático de Derecho del Trabajo y de la Seguridad Social. Departamento de Derecho de la Empresa. San Sebastián.

Universidad Autónoma de Barcelona. Catedrático de Derecho del Trabajo. Barcelona.

Universidad de Vigo. Catedrático Derecho del Trabajo y la Seguridad Social. Vigo.

Departamento de Educación Gobierno de Navarra. Profesor asignatura Formación y Orientación Laboral. Asociación Nacional de Profesorado de FOL. Pamplona.

Fundación Formación y Empleo Miguel Escalera FOREM. Presidenta confederal. Madrid. 


\section{Organización. Cargo de la persona. Ubicación}

Asociación Nacional de Agencias de Colocación. Presidencia. Madrid.

Asociación de Empresas de Trabajo Temporal y Agencias de Colocación en España ASEMPLEO. Director. Exdirector General de Empleo de la Generalitat de Catalunya. Madrid.

Servicio Público de Empleo Estatal (SEPE). Representante Subdirección General Adjunta de Políticas Activas de Empleo. Madrid.

Partido Socialista Obrero Español. Ex-Secretaria de Estado de Empleo del Ministerio de Empleo y Seguridad Social. Madrid.

Consultoría Políticas y Proyectos Sociales. Representante. Valencia.

Cáritas España. Representante. Madrid.

Fundación Secretariado Gitano. Subdirector de Programas. Madrid.

Fundación Tomillo. Representante. Madrid.

Fundación Novia Salcedo. Representante. Bilbao.

Gobierno de La Rioja. Representante Consejería de Industria y Empleo. Logroño.

Departamento de Políticas Sociales del Gobierno de Navarra. Representante. Pamplona.

Servicio Navarro de Empleo. Jefe Sección Intermediación y Orientación. Pamplona.

Instituto Aragonés de Empleo. Representante Dirección Oficina Empleo. Zaragoza.

Servicio Navarro de Empleo. Orientador nivel A. Pamplona.

Confederación Empresarios de Navarra. Representante. Pamplona.

Entidad 1 Tercer Sector de Acción Social Navarra. Representante. Pamplona

Entidad 2 Tercer Sector de Acción Social Navarra. Representante. Pamplona.

Entidad 3 Tercer Sector de Acción Social Navarra. Representante. Pamplona.

Universidad Pública de Navarra. Jefe Servicio de Orientación Profesional. Pamplona.

Instituto Navarro para la Formación, el Reciclaje y el Empleo. Representante. Pamplona.

Centro Europeo de Innovación y Empresa. Directora. Pamplona.

Consorcio Desarrollo Rural zona norte. Director. Navarra.

Unión General de Trabajadores Navarra. Representante. Pamplona.

Ayuntamiento Pamplona. Representante. Área Bienestar Social. Pamplona.

Técnico de Empleo y Juventud Ayuntamiento. Representante. Localidad navarra.

Ayuntamiento Pamplona. Representante Equipo Incorporación Socio Laboral. Pamplona.

Periódico autonómico. Responsable Sección Empleo y Emprendimiento. Pamplona.

SEPE Gipuzkoa. Representante. San Sebastián.

Servicio Navarro de Empleo. Directora Gerente. Pamplona.

Fuente: Elaboración propia. 


\section{Instrumento}

El instrumento clave es la propia persona investigadora, encargada de obtener percepciones, discursos, vivencias, y formas de expresión. El autor de la investigación se introdujo en la atmósfera que rodea al sistema de la orientación, recogió los datos y las unidades de análisis, y los analizó convenientemente. Las principales herramientas han sido tres. Primeramente, la documentación analizada (libros, artículos, informes, etc.). Seguidamente, el cuaderno de bitácora (de campo, y de análisis). Finalmente, la entrevista semiestructurada como herramienta clave (Kvale, 2011), el diseño de esta principal herramienta partió de un primer guion alineado con el principio de anticipación, al tomar en consideración la posterior etapa de análisis, teniendo en cuenta los cinco grandes códigos (categorías a priori) establecidos en la investigación.

- Razones del papel actual de la orientación las políticas de empleo.

- Valoración del grado de integración e interconexión en los sistemas de orientación.

- Limitaciones que presenta esta disciplina en materia de política de empleo.

- Tratamiento de la evaluación de las medidas de orientación.

- Opiniones sobre el futuro de la orientación en materia de política de empleo.

- Se buscó la calidad en la investigación, de forma que el rigor se asocie con la solidez del proceso investigador. Cuidando los criterios de: indicación y adecuación del diseño; diversidad de voces, inclusividad y validez democrática (Tashakkori \& Teddlie, 2003); dependencia (Creswell, 2009); credibilidad (Sabirón, 2006); y calidad de las entrevistas realizadas. Las 360.630 palabras recolectadas en el transcurso de las 52 entrevistas aportan una cantidad de respuestas espontáneas, ricas, específicas y pertinentes (Flick, 2015) (Ver Tabla 2).

Tabla 2

Guion de entrevista

Preguntas realizadas

1.- ¿Cómo valoraría el desarrollo experimentado por los sistemas de orientación profesional en los últimos años en España?

2.- ¿Cómo entender el estatus otorgado a la orientación profesional en las políticas laborales actuales?

3.-Las instituciones vienen hablando de nueva cultura de empleo. ¿Qué opinión le merece la labor que puede desarrollar las medidas de orientación en dicha nueva cultura?

4.-El concepto de flexiguridad tiene un gran eco en el mundo laboral, en línea con los mercados de trabajo transicionales actualmente vigentes. ¿Cómo considerar el papel que otorga esta modelo a orientación profesional? 


\section{Preguntas realizadas}

5.-El emprendimiento aparece como como un elemento clave en la actualidad. Por ejemplo, con la puesta en marcha de la Estrategia de Emprendimiento y Empleo Joven 2013-16. ¿Qué opinión le merece el despliegue actual de servicios, acciones y programas de orientación para el emprendimiento y autoempleo?

6.- El modelo de aprendizaje a lo largo de la vida Lifelong learning, lleva de la mano la necesidad de una orientación a lo largo de toda la vida. ¿Cómo valora el desarrollo nuestro país de esa orientación a lo largo de toda la vida?

7.- ¿Qué puede hacer la orientación profesional ante el fenómeno de la precariedad laboral?

8.- Las directrices europeas señalan la necesidad unos Servicios Públicos de Empleo que fomenten una orientación profesional sostenible para alcanzar unos resultados de activación sostenibles de los clientes. ¿Qué opinión le merece?

9.- Los principios de actuación de la Estrategia Española de Activación para el Empleo señalan la necesidad de dar un tratamiento personalizado de los demandantes de empleo. ¿Cómo valora el desarrollo de este principio en España?

10.- ¿Qué grado de cumplimiento considera usted que se está dando de los objetivos exigidos a la orientación en la política de empleo española? Reflejados en la Estrategia Española de Activación para el Empleo.

11.- ¿Cómo valora el tratamiento a la calidad en los sistemas de orientación profesional de nuestro contexto?

12.- Nuestro país ha desarrollado varias medidas dentro de las políticas de empleo: Intermediación laboral. Políticas activas de empleo, donde se encuentran las medidas de orientación. Y medidas de políticas pasivas de empleo. ¿Cómo valora el grado de integración entre ellas, por ejemplo, entre orientación profesional e intermediación laboral, o entre orientación profesional y formación para el empleo?

13.- ¿Cómo están ayudando las medias de orientación profesional a la mejorar la situación de empleo de las personas con problemáticas específicas: discapacidad, exclusión social, etc.?

14.-Las directrices europeas y estatales vienen insistiendo en la necesidad de lograr unos sistemas de orientación profesional lo más integrados posibles. ¿Qué grados de integración considera que se están dando en la actualidad?

15- La relación entre nivel educativo y empleabilidad está demostrada. En su opinión ¿Cómo podemos medir la eficacia de las medidas de orientación profesional en materia de políticas de empleo?

16.- ¿Cómo ve el futuro de la orientación profesional dentro del espacio de las políticas de empleo?

Fuente: Elaboración propia. 


\section{Procedimiento}

Las entrevistas se realizaron entre diciembre 2014 y enero 2016. Durante todo ese tiempo se procedió paralelamente a la recolección y análisis de datos cualitativos. El instrumento de registro fue la grabación de todas las entrevistas, lo que facilitó el posterior tratamiento de dichos archivos digitales. En total se sumaron 2946 minutos de grabaciones, algo más de 49 horas.

Simultáneamente al proceso de recogida de datos, se les dio estructura, organizando segmentos de información en unidades de análisis, códigos y categorías. Comprendiendo el contexto que rodea a los datos, y dándoles sentido. La inversión inicial sirvió para detectar conceptos clave y hacer emerger categorías más complejas que las cinco planteadas a priori. La inmersión profunda acometida en esta fase de trabajo de campo y análisis cualitativo dio forma a nuevos significados, relaciones y principios de teoría.

El investigador se auxilió del programa Atlas.ti 7.5.12., un programa encaminado a facilitar la aplicación de los principios metodológicos de la Teoría Fundamentada, facilitando la codificación de primer nivel y de segundo nivel.

Primeramente, la codificación de primer nivel, con la realización previa de la preparación y revisión de datos, descubriendo las unidades de análisis. Para ello el contenido de cada una de las 52 grabaciones (archivos AMR) se transcribió a archivo Word de texto, por orden cronológico, para facilitar el nivel textual de análisis con Atlas.ti. Analizándose un total de 360630 palabras, 1195 folios A4. En este nivel se creó la Unidad Hermenéutica (tesis), se trabajó con los textos, asignando los documentos primarios a dicha Unidad Hermenéutica. Se crearon cinco códigos "a priori" (free codes) como lista preliminar de categorías de análisis. Posteriormente, en este primer nivel de codificación (codificación abierta) se procedió al paciente trabajo de creación de 1052 citas "quotations" codificándolas en función de los cinco códigos creados a priori. Así mismo, a través del método comparativo constante se asignaron también a las nuevas categorías emergentes del análisis de datos asociados a fragmentos de texto (open coding); fruto de esta labor emergieron 27 sub-códigos. El proceso finalizó con la consideración del investigador de la saturación de categorías con estos 27 nuevos subcódigos bajo el paraguas de los cinco grandes códigos mencionados anteriormente. Paralelamente, de forma inductiva se fueron creando memos, anotaciones independientes de utilidad para avanzar en el proceso de codificación.

Seguidamente, la codificación de segundo nivel. De carácter más abstracto, dio significado a las categorías más avanzadas en los pasos anteriormente descritos. Sobre la cantidad de datos cualitativos recopilados, segmentados en unidades básicas, y estructurados en cinco grandes códigos en los que se fueron ordenando 27 sub-códigos, se procedió a una codificación de segundo nivel de tal forma que se fueron reduciendo los sub-códigos a los temas más significativos, reduciendo así la redundancia de categorías. El programa Atlas.ti fue de gran utilidad para establecer los diferentes tipos de relaciones entre los componentes, facilitando la creación de vistas de network (vistas de red). Generándose cinco redes focalizadas por cada uno de los cinco grandes códigos (network vie won) y una red global que sintetiza la teoría emergente (network). 


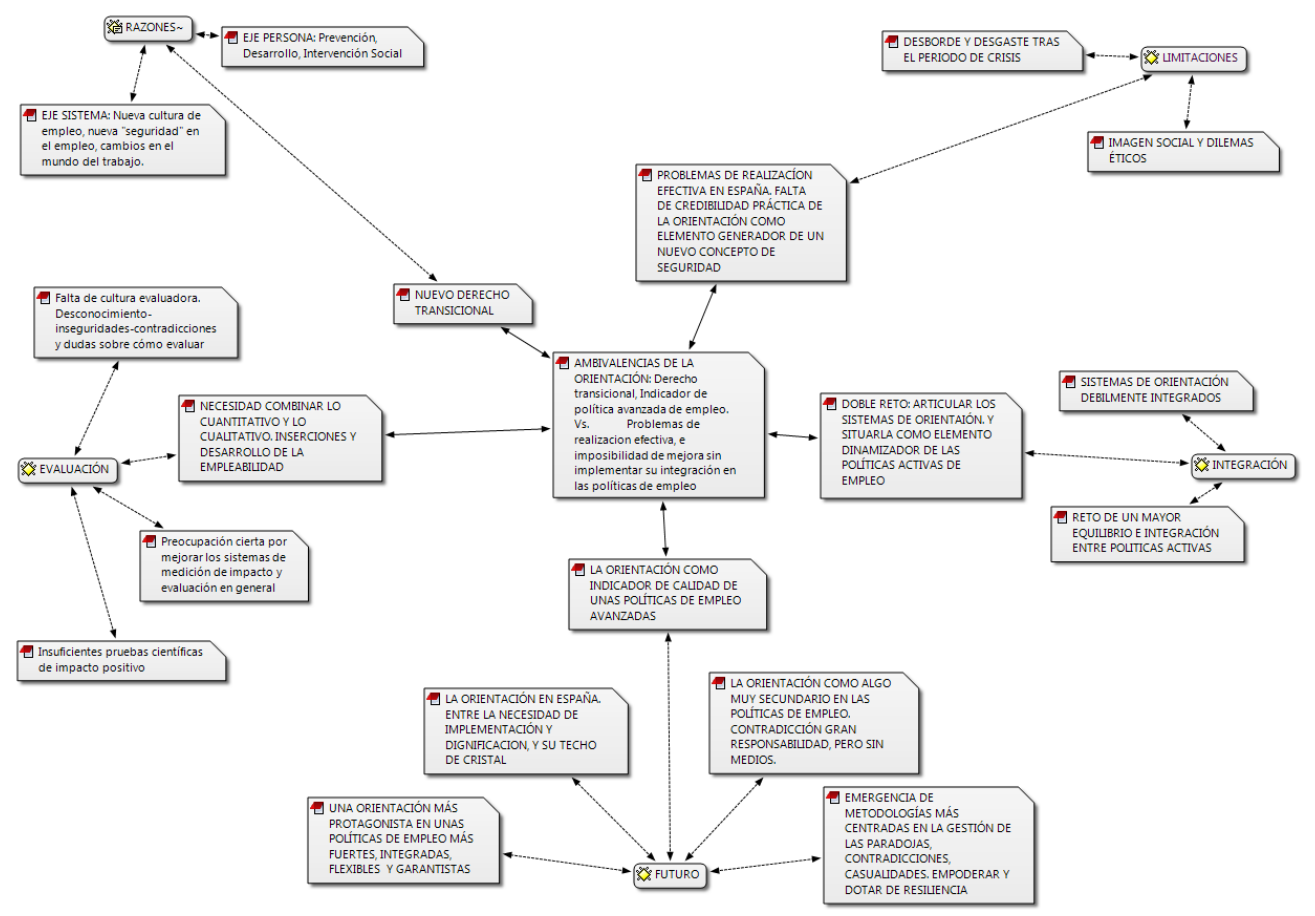

Figura 1. Red global network generada con el programa Atlas.ti. Elaboración propia

\section{Resultados}

Los cinco códigos a priori seleccionados, alineados con los objetivos de la investigación, congregaban el total de 1052 citas creadas. En primer lugar, el código sobre las razones y justificación de la implementación de la orientación en las políticas de empleo (23\% de citas). Los principales sub-códigos que emergieron señalaban: El papel de prevención y desarrollo personal, junto a la necesidad de acompañamiento. El papel de la orientación en la construcción de una nueva cultura de empleo, caracterizada por el modelo de activación, el desarrollo de la empleabilidad, y el paradigma de flexiseguridad.

En segundo lugar, el código sobre las limitaciones de la orientación fue el que más citas agrupó (40\% de citas). Emergiendo como más destacados los sub-códigos acerca de: Ausencia de una estrategia de global de fondo en orientación. La afección de debates transversales (conflictos políticos y de transferencias, relación de lo público con lo privado, desajustes entre competencias de la población y las exigencias del mundo productivo, y ambivalencias ante el fomento del emprendimiento). Los problemas de realización efectiva de la orientación en el marco laboral español. Y el desborde del sistema de orientación ante el modelo de flexiseguridad español.

En tercer lugar, el código de integración de la orientación (12\% de las citas). Que señala la complejidad de la integración e interconexión en el propio seno del sistema 
de orientación profesional. Además de las complejidades de la integración de la orientación con el resto de políticas activas de empleo. Y la integración de la orientación en relación entre políticas activas y pasivas.

En cuarto lugar, el código sobre el tratamiento de la evaluación a las medidas de orientación en política de empleo (12\% de las citas). Hace emerger de forma destacada las dificultades de dicha tarea, y la significativa cacofonía de voces y dudas al respecto.

Finalmente, en quinto lugar, el código acerca del futuro (11\% de citas). Señala el paso de una visión optimista asociada a unas necesarias propuestas de mejora del sistema. Observa opiniones de carácter más neutro, y opiniones claramente disconformes y críticas con el panorama presente y futuro.

Toda esta amplia estructura de códigos, sub-códigos y temas emergentes resultantes de la investigación se muestra en la siguiente la tabla 3.

Tabla 3

Estructura de códigos, subcódigos, y temas emergentes en la investigación.

\section{Razones de la orientación. 246 citas. $23 \%$ del total}

Prevención, desarrollo, acompañamiento

Apoyo psicológico, recomponer a la persona

Lograr mantenimiento en el mercado laboral

Lucha contra la exclusión social

Dotar de realismo, situar a la persona

Potenciar el desarrollo de las personas

Trabajar la motivación, empoderar

Traje a medida de cada persona, tratamiento individualizado

Nueva cultura de Empleo: Activación, emple- Paradigma de activación

abilidad, flexiseguridad

Movilización de subjetividades

Necesidad de conectar políticas pasivas con activas

Mejora de la Empleabilidad como referente

Desarrollo competencial

Aumento de la presión por la adaptabilidad y

la flexibilidad de las personas

Encaje de la orientación en el paradigma de

flexiseguridad

Derecho transicional

Debilidad de la orientación en ese intercam-

bio real de flexibilidad-seguridad 
Planificar el desarrollo humano de una sociedad

Orientación al autoempleo y emprendimiento como oportunidad

Modernización de las políticas activas de empleo

Impacto de la Globalización en nuestras relaciones laborales
Mejores decisiones para una mejor gestión de carrera profesional Orientación como dinamizadora del triángulo empleo-formacióneducación

Terapia de choque ante desajustes serios entre competencias y demandas del mercado

Mirada beneficio a largo plazo, ante miradas más a corto plazo

La orientación como PAE no excesivamente erosionada

Necesidad de implementar el espíritu emprendedor

Fomento del intra-emprendimiento

Emprendimiento como una oportunidad, respuesta a necesidades sociales

Influencia clara de los mandatos de la UE

Indicador de una moderna política de empleo

La orientación como vertebradora de las políticas de empleo

Elemento reductor de los desajustes entre oferta y demanda

Medidas sociolaborales correctoras del impacto de la globalización

Aceleración de los cambios en el mundo del trabajo

Demanda de nuevas competencias laborales

Nuevo modelo, respuestas colectivas vs. respuestas individuales

Adaptabilidad y flexibilidad

Nuevas formas de nombrar a la seguridad en el empleo, y a la inseguridad

Trabajo psicológico y de Orientación situada claramente en el eje sistema, ante el eje perlas subjetividades sona

El problema ya no es social o político, es de uno mismo

Medida de choque ante la crisis

Con las crisis gana peso la orientación

Aprovechar los pocos espacios que ofrece el mercado laboral

Mejora de empleabilidad para cuando mejoren las oportunidades

Mínima respuesta, por imagen, de la Administración

Principio de interven- Orientador como agente de cambio

ción social
No excluir a nadie

Derechos laborales de las personas

Repartir responsabilidades, no solo las individuales, sino las colectivas

El centro en la persona 


\section{Limitaciones de la orientación. 420 citas. $40 \%$ del total}

Sin estrategia global de Un caballo de batalla de toda la vida fondo

Una asignatura pendiente

Interés por la orientación en épocas de decrecimiento económico, desinterés en épocas de crecimiento económico

Insuficiente voluntad política para desarrollar y sostener políticas de orientación

Carácter reactivo de la orientación, ante la necesidad de prevención

Intermitencias de los servicios

Retraso estructural de los servicios públicos de empleo

Orientación ante cuatro Contacto con el mundo político

debates transversales:

Relación entre lo público y lo privado

Desajuste entre el nivel competencial real y las competencias demandas

Posicionamientos ante el emprendimiento

Problemas de realización efectiva de la orientación

Imposibilidad de garantizar un tratamiento personalizado, problemas presupuestarios

Desequilibrios internos dentro de las políticas activas de empleo Inflamación del gasto en incentivos a la contratación

Desborde del sistema de orientación. Contexto precario. Modelo de flexiseguridad español

Ante determinadas tasas de desempleo la orientación tiene poco que hacer

Relatividad de las posibilidades de las Políticas Activas de Empleo Características precarias del empleo que se genera en España Mercado laboral segmentado Realidad de los trabajadores pobres Enormes dificultades para los mayores de 45 años Falta de credibilidad del modelo teórico válido de flexiseguridad cuando se observa su puesta en práctica en España Se adquiere solo una parte de la realidad de lo que se hace en países como Dinamarca 
Imagen social y dilemas Desplazamiento de las responsabilidades sociales colectivas, a las éticos de la orientación responsabilidades individuales

Uso perverso de la orientación si no se tiene a la persona en el centro

Valoración acrítica de las dinámicas del mercado laboral

Situación compleja de actuación ante personas perceptoras de rentas

La orientación como elemento placebo y de compra de paz social

Cumplir objetivos a toda costa, para garantizar el acceso a los fondos

Imagen de la figura de orientador laboral

Desgastada. Imagen de la orientación, ante sus problemas de realización efectiva.

Imagen no óptima de la orientación laboral

Insuficiencias:

metodológicas, de competencias de los profesionales, y de atención académica
Insuficiente atención de determinados mundos académicos, como el del derecho del trabajo

Dudas acerca de las metodologías más adecuadas que requiere el momento

Nivel competencial de los profesionales de la orientación no es el adecuado en muchos casos

\section{Integración de la orientación. 125 citas. $12 \%$ del total.}

Integración del propio

sistema de orientación

Integración de la orientación con el resto de las políticas activas de empleo
Descripción de la realidad; muy pobre

Posiciones que niegan la existencia de verdaderos sistemas de orientación Asignación de responsabilidades

Aspectos y pasos positivos.

Distancia empleo educación y formación

Débil interconexión entre ejes-medidas de políticas activas

Debates Estado-Autonomías complejos

Más intensidad conforme aumenta el desempleo

Necesidad de mejoras evidentes

Reconocimiento de avances

Relación pendular entre orientación e intermediación

La relación entre orientación y FP para el empleo

Orientación e incentivos a la contratación. 
Integración de la ori- Activación de personas perceptoras de personas perceptoras

entación en la relación Área donde más queda por hacer en integración

entre políticas activas y

con políticas pasivas

Área sensible. Serios disensos.

Evaluación de la orientación. 144 citas. $12 \%$ del total

Diversidad de propues- Amplitud y desorden de propuestas, desorientación

tas- Dificultades para la Modelos econométricos

evaluación

Mirada a largo plazo

Evaluar en función del colectivo

Evitar el fracaso

Orientación a resultados

Complementar lo cuantitativo con lo cualitativo

Técnicas contrafactuales

Grupo control

Grupo de referencia

Necesidad de evaluar el impacto de las políticas

Ausencia de cultura evaluadora

Complejo encaje en un mercado laboral fracturado

Insuficientes evidencias de impacto positivo

Mejor no evaluar en exceso

Influencias en España de elementos informales de peso

Insuficientes investigaciones

Rigideces del Sistema Nacional de Empleo

Excesiva presión por evaluar.

Valoraciones del trata- Diferentes estándares bajo un mismo concepto

miento de la calidad Elemento básico

Parafernalia

Realidad pobre

Necesidad de una mirada más macro, y tan micro.

Acento en el desarrollo Desarrollo de la empleabilidad

competencial y de la

empleabilidad

Orientación por competencias.

Valoraciones del grado de cumplimiento de los

Cambio sustancial

objetivos de la orien-

tación

Si el objetivo es fomentar la adaptabilidad, éxito. 

Acento en la inserción
Acento en la inserción laboral
en el empleo
Debate eterno sobre qué es inserción

Nota. Elaboración propia.

\section{Discusión y conclusiones}

La investigación refleja el estado de opinión acerca de la realidad de la orientación en las políticas de empleo españolas en un momento crucial, el periodo 2013-2016, periodo que comprende desde el cénit de la crisis hasta la situación de postcrisis. Fruto del análisis de los resultados emerge un papel de la orientación en política de empleo de carácter oficialista, asentado normativamente pero insuficiente implementado, desbordado por momentos ante las cifras de desempleo, solapado por el protagonismo y peso presupuestario de otras políticas activas de empleo. Los logros de la investigación se sintetizan en este apartado.

Se detecta un amplio abanico de discursos que justifican el desarrollo de esta disciplina bajo dos grandes miradas: una primera educativa, donde las medidas de orientación se alinean con los principios básicos de prevención, desarrollo e intervención social, observándose una minoración del principio de intervención social (Sultana, 
2016); una segunda económico-laboral, donde emerge una orientación alineada con las trasformaciones del mundo laboral y las distintas subjetividades para adaptarse a sus dinámicas. Así la orientación se sitúa entre el paradigma de activación y el paradigma de flexiseguridad. Aparece nítidamente como seña de la modernización de los sistemas nacionales de empleo, con la aspiración de una mejor gestión de las carreras. Emerge en el periodo estudiado una orientación en España a veces reactiva, cortoplacista, como medida de choque ante la crisis de empleo (Fernández Rodríguez \& Serrano, 2014).

Se detecta una demanda de mejora global de los niveles de integración en las medias de orientación. Destacan algunos señalamientos normativos en favor de Sistemas Integrados de Información y Orientación (Echeverría \& Martínez Clares, 2015), si bien se detecta la timidez de su desarrollo, unido a voces críticas que alertan del empeoramiento de esta cuestión y la débil respuesta de la Administración en el periodo estudiado. Aunque la orientación supone el primero de los ejes de política activa de empleo, tiene pendiente ganar posicionamiento ante el resto de las medidas de políticas activas de empleo. Así mismo, emerge un elemento sensible, la relación entre políticas activas y políticas pasivas, con evidentes disensos ante el nivel más adecuado de cobertura ante las situaciones de desempleo, y señalándose este terreno como el que menor integración actualmente presenta, y como un territorio donde la orientación está llamada a desempeñar un mayor papel futuro, tanto desde las dinámicas de los mercados de trabajo transicionales, como desde los modelos de activación inclusiva (Zalakain \& Barragué, 2017).

El desarrollo de esta disciplina en el marco de las políticas de empleo presenta evidentes áreas de mejora, más evidentes en el alargado periodo de crisis iniciado en 2008. Todo ello hace que la orientación haya presentado a veces serios problemas de realización efectiva, especialmente las dificultades de hacer realidad el mandato del principio de tratamiento individualizado. En el modelo teórico de intercambio entre flexibilidad y seguridad en el empleo, la orientación aparece como un elemento de posible seguridad aún débil en el marco español. Esta situación se suma a las dificultades de asentar y proyectar una estrategia en el largo plazo, lo que fomenta el repetido uso de medidas de carácter reactivo. Al margen del comprensible gran peso de los presupuestos dedicados a las políticas pasivas, el fomento y bonificación de la contratación parece acaparar grandes cifras que se señala bien pudieran suponer una excelente oportunidad para mejorar significativamente el sistema de orientación en español. Finalmente, se detectan dudas sobre la credibilidad e imagen del propio sistema de orientación, junto a dilemas éticos, en relación a su capacidad de actuación y respuesta ante el exceso de demandas que impone el contexto laboral (Artiaga, Martín \& Serrano, 2014).

Es amplia la necesidad de seguir mejorando nuestra cultura evaluadora en general, y en este ámbito de política de empleo en particular, si bien es cierto que se observa la toma en consideración cada vez mayor por la Administración y entidades proveedoras de servicios. Destaca el carácter eminentemente cuantitativo de las evaluaciones actuales, junto con el aumento de la presión para el cumplimiento de objetivos. La búsqueda de mejores formas de evaluar y medir el impacto presenta muchos interrogantes en el sistema, el origen de esas dudas parece ser la necesidad de unas bases más sólidas del sistema de orientación ante las dinámicas laborales españolas, unido al riesgo de mirar 
los resultados excesivamente en el corto plazo. Finalmente se hace necesario combinar las evaluaciones cuantitativas (inserciones laborales) con las cualitativas (mejora del capital humano y social), sin olvidar la necesidad de implementar los esfuerzos en investigación y evaluación global de las políticas activas de empleo.

Un aspecto a considerar en esta investigación ha sido la dificultad para poder agrupar a personas expertas en la materia, dispersas geográficamente, para desarrollar la técnica de grupos de discusión. Otra de las limitaciones está relacionada con el periodo en el que se han realizado las entrevistas (2014-2016) condicionado por las duras consecuencias de la crisis y ajustes presupuestario, lo que ha podido influir en el clima de opinión.

\section{Prospectiva de la investigación}

Sería de interés ampliar el horizonte con futuras investigaciones en esta línea, profundizando en el papel práctico de la orientación en las futuras reformas de las políticas de empleo. Observando y garantizando su potencial desarrollo como dinamizador de las políticas activas y pasivas de empleo (Manzanares \& Sanz, 2016). Sería positivo analizar la relación de las evidencias que se vayan generando sobre el impacto de las medidas de orientación profesional ante nuevos conceptos de seguridad y estabilidad en el empleo, emanados del paradigma de flexiseguridad. Así mismo, investigaciones en esta línea pueden servir para proyectar el impacto que una mejora del sistema de orientación profesional pueda llegar a tener en la mejora del sistema educativo, y sociolaboral, estudiando y mejorando el campo de las metodologías evaluativas aplicadas a las medidas de orientación profesional, en un marco de mayor rendición de cuentas. En resumen, de esta investigación emerge el interrogante en España acerca de los techos de cristal que puede tener un hipotético sistema más avanzado de orientación, en el caso de no ir de la mano de unas políticas laborales más avanzadas, tendentes a garantizar un marco de empleo decente.

\section{Referencias}

Alcadipani, R. y Hogdson, D. (2009). By any means necessary? ethnographic access, ethics and the critical researcher. Tamara: Journal of critical postmodern organization science, 7(3/4), 127-146.

Artiaga, A., Martín, M. P. y Serrano, A. (2014) Qué significa la orientación: producción política del desempleado. En C. J. Fernández Rodríguez y A. Serrano Pascual (Coords.), El paradigma de la flexiguridad en las políticas de empleo españolas: un análisis cualitativo (pp. 411-448). Madrid: Centro de Investigaciones Sociológicas.

Sultana, R. D. (2016). La movilización de los servicios de orientación profesional en torno a una agenda de justiciar socia: una prioridad para todos. En A. Manzanares y C. Sanz (Directoras), Orientación profesional. Fundamentos y estrategias (pp. 27-38). Ciudad Real: UCLM-Wolters Kluwer.

Consejo Económico y Social. (2016). Memoria sobre la situación socioeconómica y laboral. España 2015. Madrid: CES. Recuperado de http://www.ces.es/documents/10180/3933980/Memoria_Socioeconomica_CES2015.pdf 
Corominas, E. (2006). Nuevas perspectivas de la orientación profesional para responder a los cambios y necesidades de la sociedad de hoy. Estudios sobre Educación, 11, 91-110.

Creswell, J. W. (2009). Research design - qualitative, quantitative and mixed methods approaches. Thousand Oaks (EE.UU.): Sage.

Echeverría, B. (2016). Transferencia del sistema de FP Dual a España. Revista de Investigación Educativa. 34(2), 295-314. doi: 10.6018/rie.34.2.249341

Echeverría, B. y Martínez Clares, P. (2015). Luces y sombras de la orientación. Revista Interuniversitaria de Formación del Profesorado, 18(2), 1-13.

Fernández, C.J. y Serrano, A. (Coords.). (2014). El paradigma de la flexiguridad en las políticas de empleo españolas: un análisis cualitativo. Madrid: CIS.

Flick, U. (2015). El diseño de investigación cualitativa. Madrid: Morata.

García-Hernández, J.L. y Cortés, A. (2019). Análisis de la eficacia de las directivas europeas para mejorar los sistemas de orientación profesional, y su efecto sobre el empleo en la Unión Europea. Instersticios. Revista sociológica de pensamiento crítico, 13(2), 5-19.

García-Hernández, J. L. y Sánchez-Santamaría, J. (2013). Estudio descriptivo sobre los servicios de orientación para el empleo en Navarra. Revista Española de Orientación y Psicopedagogía 24(2), 37-57.

García-Hernández, J.L. (2019). El papel de la orientación profesional en las políticas de empleo españolas: Un análisis cualitativo. Zaragoza: Prensas de la Universidad de Zaragoza. Recuperado de: https://zaguan.unizar.es/record/77122

Glasser, B. G. (2014). Memoing: A vital grounded theory procedure. Mill Valley (EE.UU.): Sociology Press.

Hernández, R., Fernández, C. y Baptista, P. (2010). Metodología de la investigación (Quinta edición). D.F. (México): McGraw-Hill.

Instituto Nacional de Estadística. (2017). Encuesta de población activa. Madrid: INE. Recuperado de https://www.ine.es/dyngs/INEbase/es/operacion.htm?c=Estadistica _C\&cid=1254736176918\&menu=ultiDatos\&idp=1254735976595

Kvale, S. (2011). Las entrevistas en investigación cualitativa. Madrid: Morata.

Manzanares, A. y Sanz, C. (Coords.) (2016). Orientación profesional. Fundamentos y estrategias. Madrid: UCLM-Wolters Kluwer.

Neuman, W. L. (2009). Understanding research. Londres: Pearson.

Organización para la Cooperación y el Desarrollo Económicos. (2004). Orientación profesional y políticas públicas. Cómo acortar distancias. París: Ministerio de Educación y Ciencia. Recuperado de http://www.oecd.org/education/innovation-education/34529291.pdf

Real Decreto 1032/2017, de 15 de diciembre, por el que se aprueba la Estrategia Española de Activación para el Empleo 2017-2020. Boletín Oficial del Estado, España, 16 de diciembre de 2017.

Sánchez García, M. F. (Dir.) (2017). Orientación profesional y Personal (Segunda Edición). Madrid: Universidad Nacional de Educación a Distancia.

Sabirón, F. (2006). Métodos de investigación etnográfica en ciencias sociales. Zaragoza: Mira Editores.

Sobrado, L.M. y Cortés, A. (Coords.) (2009). Orientación Profesional: Nuevos escenarios y perpectivas. Madrid: Biblioteca Nueva. 
Sultana, R. D. (2016). La movilización de los servicios de orientación profesional en torno a una agenda de justiciar socia: una prioridad para todos. En A. Manzanares y C. Sanz (Directoras), Orientación profesional. Fundamentos y estrategias (pp. 27-38). Ciudad Real: UCLM-Wolters Kluwer.

Tashakkori, A. y Teddlie, C;. (2003). Handbook of mixed methods in social \& behavioral research. Los Angeles (EE.UU.): SAGE.

Torres, C. (Coord.) (2015). España 2015. Situación Social. Madrid: CIS.

Wiltahen, T. y Tros, F. (2004). The concept of flexicurity: a new approach to regularting employment and labour markets. Transfer, 10(2), 166-186.

Zalakain, J. y Barragué, B. (2017). Repensar las políticas sociales: predistribución e inversión social. Madrid: Grupo5.

Fecha de recepción: 15 de marzo de 2018.

Fecha de revisión: 20 de marzo de 2018.

Fecha de aceptación: 12 de diciembre de 2018. 